\title{
Combined Catheter Ablation and Left Atrial Appendage Closure in Atrial Fibrillation Patients with and without Prior Stroke
}

\author{
Bin-Feng Mo $(\mathbb{D}$, Rui Zhang, Jia-Li Yuan, Jian Sun, Peng-Pai Zhang, Wei Li, Mu Chen (D), \\ Qun-Shan Wang $\mathbb{D}$, and Yi-Gang Li $\mathbb{D}$
}

\begin{abstract}
Department of Cardiology, Xinhua Hospital Affiliated to Shanghai Jiao Tong University School of Medicine, \#1665 Kong Jiang Road, Shanghai 200092, China
\end{abstract}

Correspondence should be addressed to Qun-Shan Wang; wangqunshan@xinhuamed.com.cn and Yi-Gang Li; liyigang@ xinhuamed.com.cn

Received 12 August 2021; Revised 15 November 2021; Accepted 17 November 2021; Published 8 December 2021

Academic Editor: Joseph Dens

Copyright $\odot 2021$ Bin-Feng Mo et al. This is an open access article distributed under the Creative Commons Attribution License, which permits unrestricted use, distribution, and reproduction in any medium, provided the original work is properly cited.

\begin{abstract}
Background. Combined atrial fibrillation (AF) ablation and left atrial appendage closure (LAAC) has been practiced for management of both the symptoms and the high stroke risk of AF. Data of the combined procedure in selected patients with prior stroke are limited. The aim of this study is to compare the safety and efficacy of combined catheter ablation and LAAC between AF patients with and without prior stroke. Methods and Results. This retrospective study enrolled 296 patients who underwent combined procedures of AF ablation and LAAC. Patients were divided into two groups: 81 patients with prior stroke (Stroke group) and 215 patients without prior stroke (Control group). Combined procedures were successfully performed in all the patients. Patients in the Stroke group had higher $\mathrm{CHA}_{2} \mathrm{DS}_{2}$-VASc scores $(4.9 \pm 1.2$ vs. $3.2 \pm 1.0, P<0.001)$ and higher HAS-BLED scores $(3.5 \pm 1.1$ vs. $3.0 \pm 1.0, P<0.001)$ compared with those in the Control group. Procedure-related complications in the Stroke group included two pericardial effusions and two groin hematomas, which did not differ significantly fromthe Control group ( $4.9 \%$ vs. $4.2 \%, P=0.778)$. After a mean follow-up of 20 months, the AF-free rate of the Stroke group was comparable with that of the Control group $(64.2 \%$ vs. $68.4 \%, P=0.495)$. The relative risk reductions in stroke and bleeding (observed rate compared to that predicted from the $\mathrm{CHA}_{2} \mathrm{DS}_{2}$-VASc and HAS-BLED scores) were $80 \%$ and $79 \%$, respectively, in the Stroke group, and $62 \%$ and $62 \%$, respectively, in the Control group. Conclusions. The combination of catheter ablation and LAAC is safe and efficient in selected AF patients with prior stroke. It was observed that patients with prior stroke may benefit more from risk reductions of stroke and bleeding following the combined procedure.
\end{abstract}

\section{Introduction}

Atrial fibrillation (AF) is the most common atrial arrhythmia. One of the most severe complications of AF is ischemic stroke, and stroke caused by $\mathrm{AF}$ is associated with a poor prognosis [1]. Catheter ablation is an effective treatment for patients with symptomatic drug-refractory AF, but its role in long-term stroke prevention has not been well established [2]. Left atrial appendage closure (LAAC) has been proven as a safe and effective alternative to long-term anticoagulation in patients with an increased risk of stroke and bleeding [3]. Recently, the combined procedure of ablation for $\mathrm{AF}$ and LAAC for stroke prevention has attracted increasing attention in selected patients with symptomatic AF and a high risk of stroke. Several studies have reported the safety and feasibility of performing the combined procedure [4-7].

Patients surviving an initial stroke are at a significantly increased risk of further strokes compared to the general population. A few studies have reported the outcomes of either catheter ablation [8-10] or LAAC $[11,12]$ in patients with prior stroke. However, evidence is scarce with respect to the combined therapy of AF ablation and LAAC in patients with prior stroke. Thus, the aim of this study was to 
investigate the safety and efficacy of combined catheter ablation and LAAC in AF patients with prior stroke.

\section{Methods}

2.1. Study Population. This single-center retrospective study enrolled consecutive patients with nonvalvular AF who underwent a combined procedure of catheter ablation and LAAC between April 2017 and February 2019. All participants were included based on the following criteria: age $>18$ years; symptomatic nonvalvular AF refractory to antiarrhythmic drugs; and with $\mathrm{CHA}_{2} \mathrm{DS}_{2}$-VAS score $\geq 2$ plus one of the following situations: (1) high bleeding risk (HAS-BLED score $\geq 3$ ); (2) history of stroke or systemic embolic event under oral anticoagulation (OAC) treatment; (3) intolerance to chronic $\mathrm{OAC}$; and (4) preference for LAAC device implantation as an alternative to long-term $\mathrm{OAC}[2,13,14]$. The exclusion criteria included valvular heart disease, previous $\mathrm{AF}$ ablation, recent myocardial infarction, and stroke within three months.

A total of 296 patients were enrolled. The cohort was divided into two groups: patients with prior stroke (the Stroke group, $n=81$ ) and patients without prior stroke (the Control group, $n=215)$. This retrospective study was approved by the Ethics Committee of Xinhua Hospital Affiliated to Shanghai Jiao Tong University School of Medicine and complies with the Declaration of Helsinki. Written informed consent was obtained from each patient.

2.2. Preprocedural Assessment. All the procedures were performed in a high-volume AF center ( $>1000$ cases of AF intervention per year) and undertaken by experienced operators who had passed the learning curves of either catheter ablation or LAAC. Left atrial appendage thrombus exclusion and size measurement were conducted by transesophageal echocardiography (TEE) before procedures. A cardiac computed tomography (CT) scan and 3-dimensional reconstruction of the left atrium were performed preprocedurally in 93.6\% (277/296) of patients to assist catheter ablation and LAAC.

2.3. Combined Procedure. The combined procedure was performed as described previously [7]. AF ablation was performed before LAAC implantation. Under conscious sedation, a decapolar catheter was positioned in the coronary sinus and two transseptal accesses were obtained through the right femoral vein. Mapping and ablation were performed either under the guidance of CARTO (Biosense Webster, Diamond Bar, CA, USA) or Ensite (St. Jude Medical, St. Paul, MN, USA) 3-dimensional electroanatomic mapping systems. For patients with paroxysmal AF, standard pulmonary vein isolation was performed, and for those with persistent $\mathrm{AF}$, additional linear and/or complex fragmented atrial electrogram ablations were performed according to the physician's discretion. Sinus rhythm was restored by either ablation or electric cardioversion.

LAAC was performed after AF ablation. The LAAC procedure was performed as described previously $[15,16]$. In brief, the LAAC procedure was performed under local anesthesia and fluoroscopy guidance, and TEE was introduced under deep sedation after device deployment to reconfirm the position of the device before release. A mean left atrial pressure of above $10 \mathrm{mmHg}$ was obtained after transseptal puncture. A WATCHMAN device (Boston Scientific, Marlborough, MA, USA) with an appropriate size (21, 24, 27, 30, and $33 \mathrm{~mm}$ ) was chosen, generally, 10-30\% oversizing based on the ostial width of the LAA measured by angiography or cardiac CT. The device was then advanced into the delivery sheath and deployed by sheath retraction guided by fluoroscopy. A preliminary assessment was performed by angiography and tug test under fluoroscopy to check the device position and stability. TEE was then performed to reconfirm the position with minimal $(<5 \mathrm{~mm})$ to no residual peridevice leaks and a proper compression ratio under deep sedation. The device was released if it was verified by the assessment of "PASS" criteria.

2.4. Postprocedural Anticoagulation. Patients received OAC therapy for at least 3 months following the procedure, unless there were contraindications. Dual antiplatelet therapy was recommended for another 3 months, and then life-long aspirin was prescribed if follow-up TEE showed either complete closure of the LAA or limited residual peridevice flow (jet $<5 \mathrm{~mm}$ in width).

2.5. Follow-Up. After discharge, office or transtelephonic visits were scheduled for the 3rd month, 6th month, and 12th month following the procedure and once every half a year thereafter. ECG or $24 \mathrm{~h}$ Holter monitoring was performed at each office visit for patients. Antiarrhythmic drug therapy was discontinued after 3 months if no clinical or documented AF recurrences were identified. TEE was performed to assess the device occlusion safety and efficiency at 45 days of follow-up time points. Adverse events were reported during the follow-up visit, based on the percutaneous LAA occlusion Munich Consensus Document [14], including mortality, thromboembolic events (stroke and systemic embolism), and bleeding events.

2.6. Statistical Analysis. Continuous variables are described as mean \pm standard deviation (median (interquartile range) for nonnormal data) and are compared using the Student's $t$-test (Mann-Whitney $U$ test if normality is not satisfied). Categorical variables are presented as percentages and are analyzed using the chi-square test or Fisher exact test where appropriate. The observed stroke and bleeding event rates during follow-up are calculated as the number of events per 100 patient-years and are compared with the predicted event rates based on the CHA2DS2-VASc and HAS-BLED scores using published literature [17, 18]. All analyses were performed using SPSS version 22.0 (IBM Software Inc., Armonk, NY). Two-sided $P$ values of $<0.05$ were considered statistically significant.

\section{Results}

3.1. Baseline Characteristics. A total of 81 patients in the Stroke group and 215 patients in the Control group were included. Mean ages were $69.6 \pm 8.2$ years and 68.9 \pm 7.9 
years in the two groups (Table 1). Indications for LAAC are demonstrated in Table 1. Patients in the Stroke group had a higher risk for stroke based on the $\mathrm{CHA}_{2} \mathrm{DS}_{2}$-VAS score $(4.9 \pm 1.2$ vs. $3.2 \pm 1.0, P<0.001)$ and had a higher bleeding risk based on the HAS-BLED score (3.5 \pm 1.1 vs. $3.0 \pm 1.0$, $P<0.001)$ compared with those in the Control group. The other baseline characteristics were comparable between the two groups and are described in Table 1.

3.2. Procedural Characteristics. The periprocedural outcomes are given in Table 2. The procedure time $(154.1 \pm 24.9 \mathrm{~min}$ vs. $159.7 \pm 28.6 \mathrm{~min})$ and fluoroscopy time $(10.2 \pm 3.2 \mathrm{~min}$ vs. $10.9 \pm 3.4 \mathrm{~min})$ were comparable between the Stroke and Control groups. A total of 37 patients (45.7\%) in the Stroke group and 106 patients (49.3\%) in the Control group underwent standard pulmonary vein isolation only $(P=0.578)$, while additional linear/CFAE ablations were performed in the rest of the patients. All patients in the two groups achieved a satisfactory seal (residual leak $\leq 5 \mathrm{~mm}$ ). Complete occlusion was achieved in $95.1 \%$ of the Stroke group and $94.0 \%$ of the Control group $(P=0.715)$.

There were 4 patients $(4.9 \%)$ in the Stroke group with procedure-related complications. Two were pericardial effusions which required percutaneous drainage, and the other two were minor groin hematomas. In the control group, four (1.9\%) had pericardial effusion and three $(1.4 \%)$ had groin hematomas. One patient (0.5\%) in the Control group suffered a transient coronary air embolism with chest pain and ST-segment elevation in inferior wall leads, which was resolved by forced coughing. Periprocedural stroke occurred in one patient in the Control group on the second day of the procedure and was confirmed by cranial CT. No significant difference was observed in the procedure-related complications between the two groups ( $4.9 \%$ vs. $4.2 \%, P=0.778)$.

3.3. Clinical Outcomes. Data on TEE imaging at least 45 days after the procedure were available in 77 patients $(95.1 \%)$ of the Stroke group and 208 patients $(96.7 \%)$ of the Control group. Eleven patients were evaluated by CT imaging. Satisfactory LAA occlusion (residual leak $\leq 5 \mathrm{~mm}$ ) was noted in all patients in the Stroke group and $99.5 \%$ of patients in the Control group $(P=0.539)$ (Table 3). One patient of the Control group had a residual leak $>5 \mathrm{~mm}$ due to device migration and was continued on OAC. Device-related thrombosis was detected on TEE in one patient $(1.2 \%)$ in the Stroke group and two patients $(0.9 \%)$ in the Control group, which resolved without clinical sequelae on continued oral anticoagulation.

The average follow-up was $20.8 \pm 7.0$ months in the patients of the Stroke group and $20.2 \pm 6.3$ months in the patients of the Control group (Table 3). A total of 52 patients $(64.2 \%)$ in the Stroke group and 147 patients $(68.4 \%)$ in the Control group were AF-free $(P=0.495)$ (Table 3). Fifteen patients (18.5\%) in the Stroke group and $32(14.9 \%)$ in the Control group who had an AF recurrence underwent a repeat ablation.

After the procedure, $93.8 \%$ of the Stroke group and $94.9 \%$ of the Control group were prescribed an OAC, while
TABLE 1: Baseline characteristics of the study population.

\begin{tabular}{lccc}
\hline & $\begin{array}{c}\text { Stroke } \\
\text { group } \\
N=81\end{array}$ & $\begin{array}{c}\text { Control } \\
\text { group } \\
N=215\end{array}$ & $P$ value \\
\hline Female & $36(44.4)$ & $107(49.8)$ & 0.414 \\
Age (years) & $69.6 \pm 8.2$ & $68.9 \pm 7.9$ & 0.476 \\
BMI $\left(\mathrm{kg} / \mathrm{m}^{2}\right)$ & $24.5 \pm 3.1$ & $24.8 \pm 3.4$ & 0.492 \\
Paroxysmal AF & $40(49.4)$ & $97(45.1)$ & 0.512 \\
Persistent AF & $41(50.6)$ & $118(54.9)$ & 0.512 \\
Coronary artery disease & $15(18.5)$ & $49(22.8)$ & 0.426 \\
Heart failure & $17(21.0)$ & $58(27.0)$ & 0.291 \\
Hypertension & $64(79.0)$ & $175(81.4)$ & 0.643 \\
Diabetes mellitus & $18(22.2)$ & $65(30.2)$ & 0.171 \\
Previous stroke & $81(100.0)$ & $0(0.0)$ & $<0.001$ \\
Left atrial diameter (mm) & $43.1 \pm 5.1$ & $42.5 \pm 5.7$ & 0.353 \\
LVEF (\%) & $62.8 \pm 6.5$ & $63.6 \pm 6.3$ & 0.359 \\
CHA ${ }_{2}$ DS ${ }_{2}$-VASc score & $4.9 \pm 1.2$ & $3.2 \pm 1.0$ & $<0.001$ \\
HAS-BLED score & $3.5 \pm 1.1$ & $3.0 \pm 1.0$ & $<0.001$ \\
Indications for LAAC & & & $<0.001$ \\
$\quad$ High bleeding risk & $40(49.4)$ & $124(57.7)$ & \\
History of stroke under & $21(25.9)$ & $0(0.0)$ & \\
OAC & & & \\
Intolerance to chronic & $7(8.6)$ & $22(10.2)$ & \\
OAC & $13(16.0)$ & $69(32.1)$ & \\
Patient preference & &
\end{tabular}

Values are mean $\pm \mathrm{SD}$ or $n(\%)$ as appropriate. AF, atrial fibrillation; $\mathrm{BMI}$, body mass index; LAAC, left atrial appendage closure; LVEF, left ventricular ejection fraction; OAC, oral anticoagulation.

the rest of the patients were given antiplatelet therapy. During the latest follow-up, 3.7\% of the Stroke group and $2.8 \%$ of the Control group remained on OAC, while antiplatelets were prescribed for $88.9 \%$ of the Stroke group ( $82.7 \%$ single and $6.2 \%$ dual) and $89.3 \%$ of the Control group (80.0\% single and $9.3 \%$ dual) (Table 3$)$. The remainder $(7.4 \%$ and $7.9 \%)$ received no therapy.

In the Stroke group, a total of two ischemic strokes and two gastrorrhagia were recorded at follow-up, resulting in an observed annualized stroke rate of $1.4 \%$ and an observed annualized bleeding rate of $1.4 \%$ (Table 3). In the Control group, five patients suffered ischemic stroke and eight had major bleeding events (gastrointestinal 4 (1.9\%), pulmonary $1(0.5 \%)$, urethral $1(0.5 \%)$, and epistaxis $1(0.5 \%)$ ), resulting in an observed annualized stroke rate of $1.4 \%$ and an observed annualized bleeding rate of $2.2 \%$. Compared with the expected stroke rate derived from the $\mathrm{CHA}_{2} \mathrm{DS}_{2}$-VASc score, an $80 \%$ annualized stroke reduction in the Stroke group and a $62 \%$ annualized stroke reduction in the Control group were observed (Figure 1). Annualized bleeding reduction of $79 \%$ in the Stroke group and $62 \%$ in the Control group was found compared to that expected from the HASBLED score (Figure 2).

\section{Discussion}

The current study presents a comparison between AF patients with and without prior stroke who underwent the combined procedures. The results demonstrate that combined therapy of catheter ablation and LAAC in selected AF patients with prior stroke is safe and efficient. Compared 
TABle 2: Procedural characteristics and safety.

\begin{tabular}{|c|c|c|c|}
\hline & $\begin{array}{c}\text { Stroke group } \\
\quad N=81\end{array}$ & $\begin{array}{c}\text { Control group } \\
\qquad N=215\end{array}$ & $P$ value \\
\hline Procedure time (min) & $154.1 \pm 24.9$ & $159.7 \pm 28.6$ & 0.123 \\
\hline Fluoroscopy time (min) & $10.2 \pm 3.2$ & $10.9 \pm 3.4$ & 0.117 \\
\hline PVI only & $37(45.7)$ & $106(49.3)$ & 0.578 \\
\hline PVI plus linear/CFAE ablation & $44(54.3)$ & $109(50.7)$ & 0.578 \\
\hline \multicolumn{4}{|l|}{ Morphology of LAA } \\
\hline Cauliflower & $48(59.3)$ & $133(61.9)$ & 0.682 \\
\hline Chicken wing & $18(22.2)$ & $40(18.6)$ & 0.485 \\
\hline Cactus & $8(9.9)$ & $27(12.6)$ & 0.524 \\
\hline Windsock & $7(8.6)$ & $15(7.0)$ & 0.626 \\
\hline LAA ostium width (mm) & $22.5 \pm 3.0$ & $23.1 \pm 3.4$ & 0.165 \\
\hline Device size $(\mathrm{mm})$ & $28.1 \pm 3.1$ & $28.6 \pm 3.4$ & 0.253 \\
\hline Device compression (\%) & $20.0 \pm 4.8$ & $19.3 \pm 5.4$ & 0.292 \\
\hline Successful implantation & $81(100)$ & $215(100)$ & - \\
\hline \multicolumn{4}{|l|}{ Peridevice leak at implantation } \\
\hline Complete occlusion of LAA & $77(95.1)$ & $202(94.0)$ & 0.715 \\
\hline Leak $\leq 5 \mathrm{~mm}$ & $4(4.9)$ & $13(6.0)$ & 0.715 \\
\hline Leak $>5 \mathrm{~mm}$ & $0(0.0)$ & $0(0.0)$ & - \\
\hline Procedure-related complications & $4(4.9)$ & $7(4.2)$ & 0.778 \\
\hline Death & $0(0.0)$ & $0(0.0)$ & - \\
\hline Pericardial effusion & $2(2.5)$ & $4(1.9)$ & 0.740 \\
\hline Coronary air embolism & $0(0.0)$ & $1(0.5)$ & 0.539 \\
\hline Stroke & $0(0.0)$ & $1(0.5)$ & 0.539 \\
\hline Major bleeding events & $0(0.0)$ & $0(0.0)$ & - \\
\hline Complications of vascular access & $2(2.5)$ & $3(1.4)$ & 0.523 \\
\hline
\end{tabular}

Values are mean \pm SD or $n(\%)$ as appropriate. AF, atrial fibrillation; CFAE, complex fragmented atrial electrogram; LAA, left atrial appendage; PVI, pulmonary vein isolation.

TABle 3: Outcomes at follow-up.

\begin{tabular}{|c|c|c|c|}
\hline & $\begin{array}{l}\text { Stroke group } \\
\quad N=81\end{array}$ & $\begin{array}{c}\text { Control group } \\
\quad N=215\end{array}$ & $P$ value \\
\hline Average follow-up (months) & $20.8 \pm 7.0$ & $20.2 \pm 6.3$ & 0.507 \\
\hline \multicolumn{4}{|l|}{ Peridevice leak at 45 days follow-up } \\
\hline Complete occlusion of LAA & $66(81.5)$ & $165(76.7)$ & 0.380 \\
\hline Leak $\leq 5 \mathrm{~mm}$ & $15(18.5)$ & $49(22.8)$ & 0.426 \\
\hline Leak $>5 \mathrm{~mm}$ & $0(0.0)$ & $1(0.5)$ & 0.539 \\
\hline Device-associated thrombosis & $1(1.2)$ & $2(0.9)$ & 0.816 \\
\hline \multicolumn{4}{|l|}{ AF-free at follow-up } \\
\hline Overall & $52(64.2)$ & $147(68.4)$ & 0.495 \\
\hline Paroxysmal AF & $28(70.0)$ & $73(75.3)$ & 0.525 \\
\hline Persistent AF & $24(58.5)$ & $74(62.7)$ & 0.636 \\
\hline Redo ablation & $15(18.5)$ & $32(14.9)$ & 0.446 \\
\hline \multicolumn{4}{|c|}{ Antithrombotic medications at latest follow-up } \\
\hline Oral anticoagulation & $3(3.7)$ & $6(2.8)$ & 0.683 \\
\hline Dual antiplatelet therapy & $5(6.2)$ & $20(9.3)$ & 0.388 \\
\hline Single antiplatelet therapy & $67(82.7)$ & $172(80.0)$ & 0.597 \\
\hline None & $6(7.4)$ & $17(7.9)$ & 0.866 \\
\hline Thromboembolic events & $2(2.5)$ & $5(2.3)$ & 0.942 \\
\hline Ischemic stroke & $2(2.5)$ & $5(2.3)$ & 0.942 \\
\hline Systemic embolism & $0(0.0)$ & $0(0.0)$ & - \\
\hline Observed annualized stroke rate (\%) & 1.4 & 1.4 & - \\
\hline Major bleeding & $2(2.5)$ & $8(3.7)$ & 0.595 \\
\hline Intracranial & $0(0.0)$ & $0(0.0)$ & - \\
\hline Gastrointestinal & $2(2.5)$ & $4(1.9)$ & 0.740 \\
\hline Pericardial & $0(0.0)$ & $0(0.0)$ & - \\
\hline Pulmonary & $0(0.0)$ & $1(0.5)$ & 0.539 \\
\hline Urethral & $0(0.0)$ & $2(0.9)$ & 0.385 \\
\hline
\end{tabular}


TABLE 3: Continued.

\begin{tabular}{lccc}
\hline & Stroke group & Control group & $N=215$ \\
\hline Epistaxis & $N=81$ & $1(0.5)$ & $P$ value \\
Observed annualized bleeding rate (\%) & $0(0.0)$ & 2.2 & 0.539 \\
Mortality & 1.4 & $2(0.9)$ & 0.816 \\
$\quad$ Cardiovascular & $1(1.2)$ & $1(0.5)$ & 0.472 \\
Noncardiovascular & $1(1.2)$ & $0(0.0)$ & - \\
Reason unknown & $0(0.0)$ & $1(0.5)$ & 0.539 \\
\hline
\end{tabular}

Values are mean $\pm \mathrm{SD}$ or $n(\%)$ as appropriate. AF, atrial fibrillation; LAA, left atrial appendage; PVI, pulmonary vein isolation; TEE, transesophageal echocardiography.

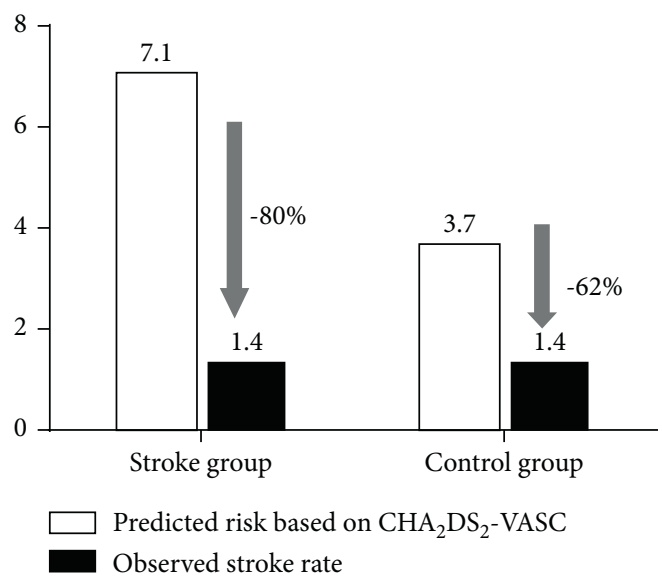

Figure 1: Efficacy in reduction of stroke rate (per 100 patientsyear) during the overall follow-up.

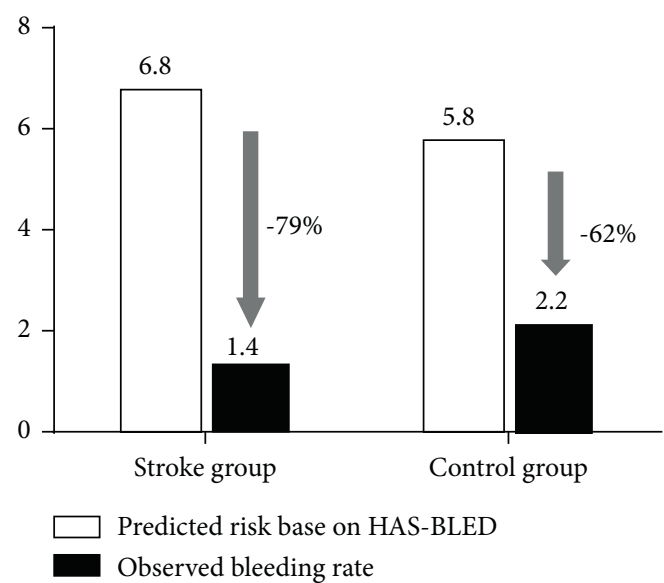

FIGURE 2: Efficacy in reduction of bleeding rate (per 100 patientsyear) during the overall follow-up.

with patients without prior stroke, patients with prior stroke may benefit more from reduced risk of stroke and bleeding following the combined procedure.

$\mathrm{AF}$ is an independent risk factor for stroke, and stroke occurring with $\mathrm{AF}$ is more likely to be fatal or more severe than non-AF stroke [19]. OAC can significantly reduce stroke events and improve outcomes in AF patients with a high risk of stroke [20]. Catheter ablation for AF has been proven to be effective in rhythm control and improves the quality of life, but no randomized clinical trial has shown a reduction in long-term ischemic stroke [21]. On the contrary, LAAC with the WATCHMAN ${ }^{\mathrm{TM}}$ device has been demonstrated in randomized trials to reduce strokes and, therefore, can be an alternative to warfarin therapy for stroke prevention [22, 23]. The combined procedure of AF ablation and LAAC can provide concomitant rhythm control as well as stroke prevention in patients with symptomatic AF and a high risk of stroke [4-7].

The safety of combined ablation and LAAC was first reported by Swaans et al. in a small observational study of 30 patients in 2012 [4]. Pulmonary vein isolation and additional complex-fractionated atrial electrogram ablation were performed, followed by LAAC with the WATCHMAN device. Successful closure with satisfactory seals was achieved in all the patients with three $(10 \%)$ patients experiencing minor periprocedural complications. During 1-year follow-up, 70\% of the patients were free from atrial arrhythmias and no thromboembolic events occurred. A high procedural success rate with a relatively low complication rate of the combined procedure as well as satisfactory midterm follow-up results was obtained in that study.

Since then, a series of observational studies, including two multicenter registry studies with long-term follow-ups, further supported and strengthened the notion that the combined therapy can be feasible, safe, and successful $[5,6]$. Wintgens et al. [5] published long-term follow-up results of a prospective real-world multicenter trial with a large patient cohort of 349 patients. Rates of satisfactory and complete LAA sealing were $100 \%$ and $92.6 \%$, respectively. After 35 months of follow-up, $49 \%$ of the patients remained AF-free. Annualized stroke and major bleeding rates were $0.7 \%$ and $1.1 \%$, respectively, referring a $78 \%$ risk reduction of stroke and a $71 \%$ risk reduction of bleeding. Phillips et al. [6] reported the long-term outcome results of 142 patients by pooling data from the EWOLUTION and WASP registries. Successful LAAC was achieved in $99.3 \%$ of the included patients, with a $97.2 \%$ complete LAA seal rate. The 30 -day device and/or procedure-related SAE rate was $2.1 \%$. After a mean follow-up of $726 \pm 91$ days, the annualized stroke and major bleeding rates were $1.09 \%$ and $1.09 \%$, respectively. We recently reported the results of the combined procedure in a case-control study using the propensity score matching method [7]. Outcomes of the combined procedure with 
ablation alone and LAAC alone were compared. Procedurerelated complications were similar among the groups. The AF-free rate was comparable between the ablation alone group and the combined procedure group (67.1\% vs. $69.7 \%$, $P>0.05)$. Complete occlusion rates were also similar between the LAAC alone group and the combined procedure group immediately postprocedure (94.7\% vs. $93.4 \%)$ and at 45 days postprocedure $(82.9 \%$ vs. $85.5 \%)$. The case-control results supplement the safety and efficacy of the combined therapy. Although the safety and efficacy of the combined procedure in ordinary patients with AF have been substantially demonstrated [4-7], evidence is scarce with respect to the feasibility of interventional therapies in $\mathrm{AF}$ patients with a previous stroke history. In this current study, we reported valuable evidence on the subgroup of patients with prior stroke and $\mathrm{AF}$ who underwent a combined procedure of ablation and LAAC.

Previous studies have reported the safety of patients with prior stroke undergoing AF catheter ablation $[8,9]$ or LAAC [11]. In the present study, we focused on selected patients with symptomatic AF and prior stroke who underwent combined therapy of catheter ablation and LAAC. We reported low periprocedural complications in patients with prior stroke which was comparable with those without prior stroke. Regarding AF recurrence, Li et al. reported a similar rate of AF recurrence between those with or without prior stroke during a near 2-year follow-up [8]. Our results demonstrated a comparable AF-free rate of $64.2 \%$ and $68.4 \%$ between patients with and without prior stroke following the combined procedure. These two consistent results suggest that a previous stroke seems not to have an impact on the success of AF ablation. Another study revealed that in patients with AF and a prior history of stroke, patients undergoing ablation have lower rates of recurrent stroke compared to AF patients not ablated with five years of follow-up [10], although the full mechanisms of benefit are not yet known.

Previous studies have shown that patients with prior cerebral embolic events are at an extremely high risk of recurrence of stroke $[24,25]$. The cumulative risk of stroke was $11.1 \%$ at 1 year and up to $26.4 \%$ at 5 years for patients after initial stroke [26]. Published studies have described the effectiveness of LAAC in patients with AF and prior stroke as secondary prevention over short-term observation [12] and long-term observation [11]. In our study, patients with prior stroke had significantly higher $\mathrm{CHA}_{2} \mathrm{DS}_{2}$-VASc and HASBLED scores than those without, which meant a higher risk of stroke and bleeding. However, the observed annualized stroke rate and annualized bleeding rate were similar in patients with and without prior stroke in this study. Thus, a more significant risk reduction of stroke and bleeding compared to that expected from the risk scores in patients with prior stroke was observed, implying that patients with prior stroke are better candidates for the combined procedure of ablation and LAAC.

There are several limitations in our study. This is a singlecenter retrospective study with a moderate sample size. The history of stroke was self-reported which may underestimate the percentage of strokes in patients with AF. Follow-up with
ECG and $24 \mathrm{~h}$ Holter recordings for detecting AF recurrence is another limitation. Asymptomatic arrhythmias or nondocumented symptomatic episodes may have been undetected.

\section{Conclusions}

In conclusion, combined therapy of AF ablation and LAAC is safe and efficient in patients with prior stroke. Long-term data and large-scale studies are needed to further verify the benefit of combined therapy in selected patients with AF and prior stroke.

\section{Abbreviations \\ AF: Atrial fibrillation \\ CT: Computed tomography \\ LAAC: Left atrial appendage closure \\ OAC: Oral anticoagulation \\ TEE: Transesophageal echocardiography.}

\section{Data Availability}

The data used to support the findings of this study are available from the corresponding author upon request.

\section{Conflicts of Interest}

The authors declare that they have no conflicts of interest.

\section{Authors' Contributions}

Bin-Feng Mo and Rui Zhang contributed equally to the study.

\section{Acknowledgments}

This work was supported by grants from the Medical-Engineering Cross Foundation of Shanghai Jiaotong University (YG2019QNA47) and Zhongnanshan Medical Foundation of Guangdong Province (ZNSA-2020017).

\section{References}

[1] C. Steger, A. Pratter, M. Martinekbregel et al., "Stroke patients with atrial fibrillation have a worse prognosis than patients without: data from the Austrian stroke registry," European Heart Journal, vol. 25, no. 19, pp. 1734-1740, 2004.

[2] P. Kirchhof, S. Benussi, D. Kotecha et al., "2016 ESC guidelines for the management of atrial fibrillation developed in collaboration with EACTS," European Heart Journal, vol. 37, no. 38, pp. 2893-2962, 2016.

[3] D. R. Holmes Jr., S. K. Doshi, S. Kar et al., "Left atrial appendage closure as an alternative to warfarin for stroke prevention in atrial fibrillation: a patient-level meta-analysis," Journal of the American College of Cardiology, vol. 65, no. 24, pp. 2614-2623, 2015.

[4] M. J. Swaans, M. C. Post, B. J. W. M. Rensing, and L. V. A. Boersma, "Ablation for atrial fibrillation in combination with left atrial appendage closure: first results of a feasibility study," Journal of the American Heart Association, vol. 1, no. 5, Article ID e002212, 2012. 
[5] L. Wintgens, A. Romanov, K. Phillips et al., "Combined atrial fibrillation ablation and left atrial appendage closure: longterm follow-up from a large multicentre registry," EP Europace, vol. 20, no. 11, pp. 1783-1789, 2018.

[6] K. P. Phillips, A. Romanov, S. Artemenko et al., "Combining left atrial appendage closure and catheter ablation for atrial fibrillation: 2-year outcomes from a multinational registry," EP Europace, vol. 22, no. 2, pp. 225-231, 2020.

[7] B.-F. Mo, J. Sun, P.-P. Zhang et al., "Combined therapy of catheter ablation and left atrial appendage closure for patients with atrial fibrillation: a case-control study," Journal of Interventional Cardiology, vol. 2020, Article ID 8615410, 8 pages, 2020.

[8] S. N. Li, J. P. Kang, X. Du et al., "Outcome of catheter ablation of atrial fibrillation in patients with prior ischemic stroke," Chinese Medical Journal, vol. 126, no. 6, pp. 1033-1038, 2013.

[9] A. A. Hussein, A. Gadre, O. M. Wazni et al., "Safety of catheter ablation for atrial fibrillation in patients with prior cerebrovascular events," Journal of the American College of Cardiology: Clinical Electrophysiology, vol. 2, no. 2, pp. 162-169, 2016.

[10] T. J. Bunch, H. T. May, T. L. Bair et al., "Five-year impact of catheter ablation for atrial fibrillation in patients with a prior history of stroke," Journal of Cardiovascular Electrophysiology, vol. 29, no. 2, pp. 221-226, 2018.

[11] R. Litwinowicz, M. Bartus, M. Malec-Litwinowicz et al., "Left atrial appendage occlusion for secondary stroke prevention in patients with atrial fibrillation: long-term results," Cerebrovascular Diseases, vol. 47, no. 3-4, pp. 188-195, 2019.

[12] Y. Chen, Y. Zhang, W. Huang, K. Huang, B. Xu, and X. Su, "Primary and secondary stroke prevention using left atrial appendage closure with watchman devices in atrial fibrillation patients: a single center experience from mainland China," Pacing and Clinical Electrophysiology, vol. 40, no. 6, pp. 607-614, 2017.

[13] M. Glikson, R. Wolff, G. Hindricks et al., "EHRA/EAPCI expert consensus statement on catheter-based left atrial appendage occlusion - an update," EuroIntervention, vol. 15, no. 13, pp. 1133-1180, 2020.

[14] A. Tzikas, D. R. Holmes Jr., S. Gafoor et al., "Percutaneous left atrial appendage occlusion: the Munich consensus document on definitions, endpoints, and data collection requirements for clinical studies," Europace, vol. 19, no. 1, 2017.

[15] B.-F. Mo, Y. Wan, A. Alimu et al., "Image fusion of integrating fluoroscopy into 3D computed tomography in guidance of left atrial appendage closure," European Heart Journal-Cardiovascular Imaging, vol. 22, no. 1, pp. 92-101, 2019.

[16] B.-F. Mo, R. Zhang, J.-L. Yuan et al., "Left atrial appendage closure for primary and secondary stroke prevention in patients with hypertrophic cardiomyopathy and atrial fibrillation: a pilot study," Frontiers in Cardiovascular Medicine, vol. 8, Article ID 719755, 2021.

[17] L. Friberg, M. Rosenqvist, and G. Y. H. Lip, "Evaluation of risk stratification schemes for ischaemic stroke and bleeding in 182,678 patients with atrial fibrillation: the Swedish atrial fibrillation cohort study," European Heart Journal, vol. 33, no. 12, pp. 1500-1510, 2012.

[18] G. Y. H. Lip, L. Frison, J. L. Halperin, and D. A. Lane, "Comparative validation of a novel risk score for predicting bleeding risk in anticoagulated patients with atrial fibrillation: the HAS-BLED (hypertension, abnormal renal/liver function, stroke, bleeding history or predisposition, labile INR, elderly, drugs/alcohol concomitantly) score," Journal of the American College of Cardiology, vol. 57, no. 2, pp. 173-180, 2011.
[19] H.-J. Lin, P. A. Wolf, M. Kelly-Hayes et al., "Stroke severity in atrial fibrillation. The Framingham study," Stroke, vol. 27, no. 10, pp. 1760-1764, 1996.

[20] C. T. January, L. S. Wann, H. Calkins et al., "2019 AHA/ACC/ HRS focused update of the 2014 AHA/ACC/HRS guideline for the management of patients with atrial fibrillation: a report of the American College of Cardiology/American Heart Association Task Force on clinical practice guidelines and the heart rhythm society in collaboration with the society of thoracic surgeons," Circulation, vol. 140, no. 2, pp. e125-e151, 2019.

[21] D. L. Packer, D. B. Mark, R. A. Robb et al., "Effect of catheter ablation vs. antiarrhythmic drug therapy on mortality, stroke, bleeding, and cardiac arrest among patients with atrial fibrillation: the CABANA randomized clinical trial," JAMA, vol. 321, no. 13, pp. 1261-1274, 2019.

[22] V. Y. Reddy, H. Sievert, J. Halperin et al., "Percutaneous left atrial appendage closure vs. warfarin for atrial fibrillation: a randomized clinical trial," JAMA, vol. 312, no. 19, pp. 1988-1998, 2014.

[23] D. R. Holmes Jr., S. Kar, M. J. Price et al., "Prospective randomized evaluation of the watchman left atrial appendage closure device in patients with atrial fibrillation versus longterm warfarin therapy: the PREVAIL trial," Journal of the American College of Cardiology, vol. 64, no. 1, pp. 1-12, 2014.

[24] J. Burn, M. Dennis, J. Bamford, P. Sandercock, D. Wade, and C. Warlow, "Long-term risk of recurrent stroke after a firstever stroke. The Oxfordshire community stroke project," Stroke, vol. 25, no. 2, pp. 333-337, 1994.

[25] K. Hardie, G. J. Hankey, K. Jamrozik, R. J. Broadhurst, and C. Anderson, "Ten-year risk of first recurrent stroke and disability after first-ever stroke in the Perth community stroke study," Stroke, vol. 35, no. 3, pp. 731-735, 2004.

[26] K. M. Mohan, C. D. A. Wolfe, A. G. Rudd, P. U. Heuschmann, P. L. Kolominsky-Rabas, and A. P. Grieve, "Risk and cumulative risk of stroke recurrence: a systematic review and meta-analysis," Stroke, vol. 42, no. 5, pp. 1489-1494, 2011. 